\title{
Escherichia coli Causing Neonatal Meningitis During 200I-2020: A Study in Eastern China
}

\author{
Yanli Liu \\ Minli Zhu \\ Xiaoqin Fu \\ Jiaojiao Cai \\ Shangqin Chen \\ Yuanyuan Lin \\ $\mathrm{Na}$ Jiang \\ Si Chen \\ Zhenlang Lin (D)
}

Department of Neonatology, The Second Affiliated Hospital and Yuying Children's Hospital of Wenzhou Medical University, Wenzhou, Zhejiang, 325027, People's Republic of China
Correspondence: Zhenlang Lin Email linzhenlang@hotmail.com
Background and Objective: Neonatal meningitis (NM) caused by Escherichia coli remains a major health problem in industrialized countries. Currently, information on the epidemiology and antimicrobial susceptibility patterns of NM in developing countries such as China is relatively scarce. Therefore, the present study investigated changes in the antimicrobial susceptibility of $E$. coli causing NM in a perinatal center in eastern China over the past 20 years.

Methods: This survey was conducted during three periods: 2001-2006, 2007-2012, and 2013-2020. NM was diagnosed according to the number of white blood cells in the cerebrospinal fluid (CSF) and the presence of a single potential pathogenic bacterium in the culture prepared from the blood or CSF of a newborn baby. Changes in the antimicrobial susceptibility of $E$. coli were analyzed.

Results: In total, 182 NM cases were identified. E. coli was identified in 69 of these cases, and in 21 of these cases, extended-spectrum beta-lactamase (ESBL) production was detected. $E$. coli was the main cause of NM identified in this study. The overall susceptibility of $E$. coli to third-generation cephalosporins such as cefotaxime decreased from $100 \%$ during $2001-$ 2006 to 50\% during 2007-2012 and, subsequently, increased to $71.0 \%$ during $2013-2020$. This pattern of change is correlated with bacterial ESBL production. Only $8.3 \%$ of E. coli found in samples collected from infants with early onset meningitis (EOM) produced ESBL, while $37.3 \%$ of $E$. coli isolated from children with late-onset meningitis (LOM) produced ESBL.

Conclusion: E. coli remains the primary pathogen of NM. Compared with that isolated from infants with LOM, the percentage of ESBL-producing multidrug-resistant $E$. coli isolated from infants with EOM is significantly lower. Clinicians should consider this trend when determining appropriate and effective antibiotics as empirical treatment for NM.

Keywords: Escherichia coli, extended-spectrum $\beta$-lactamase, meningitis, newborn

\section{Introduction}

Bacterial meningitis is related to high rates of mortality and morbidity. ${ }^{1,2}$ The mortality rates vary between $10 \%$ and $15 \%$, especially in the neonatal period. ${ }^{3,4}$ Extraintestinal pathogenic $E$. coli remains one of the most common bacterial pathogens causing extraintestinal infections including neonatal meningitis (NM), septicemia, and urinary tract infections. ${ }^{5-7}$ Early onset meningitis (EOM) is defined as the development of bacterial meningitis within 3 days of birth, while late-onset meningitis (LOM) is defined as the development of bacterial meningitis more than 3 days post-birth. ${ }^{8}$ EOM is usually acquired through vertical transmission, while LOM is generally contracted as nosocomial or community infection. ${ }^{9}$ Compared to 
LOM, infants with prolonged rupture of membranes (PROM) are more prone to develop EOM with worse outcomes because of chorioamnionitis and amniotic fluid contamination. ${ }^{10}$ E. coli is the second most common pathogen and accounts for $30 \%$ of all EOM cases in developed countries. ${ }^{11}$

Symptoms of NM are generally non-specific, for which rapid recognition and early initiation of antimicrobial therapy before the availability of blood or CSF culture results is crucial. In the 1996 national prospective study of meningitis in newborns in England and Wales, the mortality rate of $\mathrm{NM}$ in the acute stage was $6.6 \%$, ${ }^{4}$ while this rate was $22 \%$ in a similar study conducted in $1985 .{ }^{12}$ Despite the overall improvement in neonatal care from 1985 to 1996 , the primary difference between the two studies was an increase in the use of third-generation cephalosporins. ${ }^{13}$ A retrospective study conducted by Zhao et al showed that E. coli remains a prominent pathogen of NM. ${ }^{14}$ Antibiotic treatment has always been a routine treatment for this infection. However, because of the emergence of drug-resistant bacteria, the curative effects of antibiotics have decreased. Presently, E. coli has different degrees of resistance to third-generation cephalosporins. ${ }^{14}$

Although studies conducted in developed countries have reported that Group B Streptococcus (GBS), E. coli, and Listeria monocytogenes are major organisms in the spread of NM, ${ }^{15-17}$ the results from developing countries may differ. Data about the epidemiology and antimicrobial susceptibility patterns of NM in developing countries are relatively scarce, especially in China where the economy rapidly developed since the 21 st century. Almost all the reported E. coli isolates from Chinese neonates are susceptible to amikacin, cefoperazone-sulbactam, and carbapenems. $^{18,19}$ As changes in multidrug-resistant E. coli strains occur at an increasing rate globally, the spread of multidrug-resistant $E$. coli is now a public health problem and a major concerning issue in China. The present study aimed to investigate the clinical characteristics and antimicrobial susceptibility patterns of NM caused by E. coli from 2001 to 2020 in a large tertiary neonatal intensive care unit (NICU) in Wenzhou, located in the Zhejiang province of eastern China. Moreover, we compared the ratio of extended-spectrum beta-lactamase (ESBL)-producing E. coli of NM. We focused on comparing the ratio of ESBL-producing E. coli between EOM and LOM infants.

\section{Materials and Methods Data Collection}

Neonatal cases were defined as infection in infants aged $\leq$ 28 days. All newborns diagnosed with purulent meningitis in the NICU of the Second Affiliated Hospital of Wenzhou Medical University and Yuying Children's Hospital during the study periods were included in this retrospective cohort study. NM was defined by a leukocyte count $\geq 20 \times 10^{6}$ cells/L in the cerebrospinal fluid (CSF) ${ }^{20}$ and the presence of a single potential pathogenic bacterium in the culture prepared from the blood or CSF of a newborn baby. When contamination of the CSF with blood occurred during the lumbar puncture (determined by the ratio of red blood cells to white blood cells in routine blood test), white blood cells were deducted from the leukocyte count accordingly. Blood or CSF cultures positive for pathogens generally considered contaminants (eg coagulase-negative staphylococci, viridans streptococci and diptheroids, or mixed pathogens) were excluded from the positive blood and CSF results. ${ }^{20}$ Sensitivity and specificity test results and ESBL statuses were reported from our clinical laboratory, which conducts routine microbiological examinations according to the standards formulated by the American Clinical and Laboratory Standards Association. Because the present study covered an extended period, bacterial species were identified by either traditional biochemical techniques or automated methods using the VITEK system (Vitek 2 Compact, BioMerieux, France). Initially, the manual Kirby-Bauer disk diffusion method or the recent gramnegative drug sensitivity card (BioMerieux, France) was used to determine the antibiotic sensitivity of bacterial isolates. This study covered three periods: 2001-2006, 2007-2012, and 2013-2020. All cases were identified by registration and hospital diagnosis records and were confirmed by detailed chart reviews.

Relevant clinical data were extracted from patients' medical records. We collected each infant's gestational age, sex, birth weight, mode of delivery, and fever $\left(>38^{\circ} \mathrm{C}\right)$. To calculate the incidence of $\mathrm{NM}$, data of the number of total live births in the hospital during these three periods were also collected. This research was approved by the institutional ethics committee of the Second Affiliated Hospital of Wenzhou Medical College and Yuying Children's Hospital. The patients' parents provided consent to review the medical records, which was allowed by the Institutional Ethics Committee. The treatment of confidentiality of patient data strictly follows 
the rules formulated by the institution and conforms to the Helsinki Declaration.

\section{Statistical Analyses}

SPSS software (version 23.0) was used for statistical analyses. The patients' basic clinical features and blood culture results and the antimicrobial susceptibilities of their relative E. coli strains were analyzed. The Kolmogorov-Smirnov test was used to analyze the normality of continuous variables. Data with normal distribution are expressed as means \pm standard deviations and were analyzed using Student's $t$-test of variance. Data with non-normal distribution are described as medians and ranges and were analyzed using the Wilcoxon signed-rank test or the Mann-Whitney $U$-test. Classification data were analyzed using the chi-square test or Fisher's exact test. A p-value of $<0.05$ of the predicted variable was considered significant.

\section{Results}

\section{Cases of Neonatal Meningitis}

Table 1 shows the cases of neonates with NM in the three time periods. In the study hospital, the total number of live births was 17,263 during 2001-2006, 39,202 during 20072012, and 66,549 during 2013-2020. One hundred and eighty-two cases of culture-confirmed neonatal purulent meningitis were identified from 2001 to 2020, among which 69 cases of $E$. coli were isolated (including 10 cases from 2001 to 2006, 24 cases from 2007 to 2012, and 35 cases from 2013 to 2020). Of the 69 cases, 4 were isolated from blood cultures in other hospitals before being transferred to the NICU of the study hospital; therefore, no detailed information was obtained. Of the 182 infants with NM, 27 (2 from 2001-2006, 10 from 2007-2012, and 15 from 2013-2020) were born in the study hospital; the other 155 patients were either transferred from other hospitals that did not have NICU services or directly admitted from the community after birth at home to the study hospital.

Table I Cases of Neonatal E. coli Meningitis in Different Periods

\begin{tabular}{|c|c|c|c|}
\hline & $\begin{array}{l}200 I- \\
2006\end{array}$ & $\begin{array}{l}2007- \\
2012\end{array}$ & $\begin{array}{l}2013- \\
2020\end{array}$ \\
\hline Live births & 17,263 & 39,202 & 66,549 \\
\hline E. coli causing NM & 10 & 24 & 35 \\
\hline $\begin{array}{l}\text { E. coli causing NM born in the study } \\
\text { hospital }\end{array}$ & 2 & 10 & 15 \\
\hline Incidence of $E$. coli causing NM born in & $0.12 /$ & $0.26 /$ & $0.23 /$ \\
\hline the study hospital & 1000 & 1000 & 1000 \\
\hline
\end{tabular}

Therefore, the calculated incidence of culture-confirmed $\mathrm{NM}$ in the study hospital was 0.12 per 1000 live-births during 2001-2006, and 0.26 per 1000 live-births during 2007-2012, and 0.23 per 1000 live-births during 2013-2020.

\section{Bacterial Pathogens in Neonatal Meningitis}

The proportions of different bacterial pathogens that caused NM during the three time periods were compared, and the results are shown in Table 2. The proportion of GBSinduced cases of NM increased from 4.2\% during 20012006 to $17.2 \%$ during $2007-2012$ and to $40.4 \%$ during 2013-2020 $(p<0.001)$; thus, GBS became the most frequently isolated gram-positive bacteria in neonatal NM patients from 2013 to 2020 . Over the previous two decades, the proportion of NM cases attributed to $E$. coli infection remained relatively stable, remaining above $37 \%$ in each of the three periods evaluated. E. coli remained the primary bacterial pathogen conferring NM in each period. The proportion of NM cases caused by enterococcus decreased, although the differences between the three time periods were not statistically significant. Similarly, the proportion of NM cases caused by Staphylococcus aureus did not change significantly. Additionally, the proportion of Klebsiella cases also remained relatively stable, making up approximately $3 \%$ of all NM cases in every period.

\section{Clinical Characteristics of E. coli Causing Neonatal Meningitis}

Table 3 shows the general characteristics of neonates with $E$. coli NM in each of the three time periods. The proportion of infants with $E$. coli NM born at home decreased

Table 2 Distribution of Pathogens of Neonatal Meningitis in 200I-2006, 2007-2012 and 2013-2020 Were Analyzed by Pearson's Chi-Squared Test

\begin{tabular}{|l|l|l|l|}
\hline Pathogens & $\begin{array}{l}\text { 200I-2006 } \\
(\mathbf{n = 2 4 )}\end{array}$ & $\begin{array}{l}\mathbf{2 0 0 7 - 2 0 I 2} \\
\mathbf{( n = 6 4 )}\end{array}$ & $\begin{array}{l}\mathbf{2 0 I 3 - 2 0 2 0} \\
\mathbf{( n = 9 4 )}\end{array}$ \\
\hline $\begin{array}{l}\text { Gram-positive organisms } \\
\text { GBS }\end{array}$ & $\mathrm{I}(4.2 \%)$ & $\mathrm{II}(17.2 \%)$ & $38(40.4 \%)$ \\
Enterococcus & $4(16.6 \%)$ & $8(12.5 \%)$ & $6(6.4 \%)$ \\
Staphylococcus aureus & $3(12.5 \%)$ & $6(9.3 \%)$ & $3(3.2 \%)$ \\
Other & $0(0 \%)$ & $4(6.3 \%)$ & $4(4.3 \%)$ \\
\hline Gram-negative organisms & & & \\
E. coli & $10(41.7 \%)$ & $24(37.5 \%)$ & $35(37.2 \%)$ \\
Klebsiella & $\mathrm{I}(4.2 \%)$ & $2(3.1 \%)$ & $3(3.2 \%)$ \\
Other & $5(20.8 \%)$ & $9(14.1 \%)$ & $5(5.3 \%)$ \\
\hline
\end{tabular}

Notes: $* \chi^{2}=18.532, P<0.001$. 
Table 3 General Characteristics of Patients with Neonatal E. coli Meningitis

\begin{tabular}{|c|c|c|c|c|c|}
\hline & & $2001-2006(n=10)$ & $2007-2012(n=24)$ & $2013-2020(n=35)$ & $P$-value \\
\hline Male gender & 42 & $6(60.0 \%)$ & 15 (62.5\%) & $21(60.0 \%)$ & NS \\
\hline Gestational age (weeks) & & $38.2 \pm 2.8$ & $38.1 \pm 3.5$ & $36.7 \pm 3.9$ & NS \\
\hline$<37$ weeks & 17 & $2(20 \%)$ & $5(20.8 \%)$ & $10(28.2 \%)$ & NS \\
\hline Birth weight (gm) & & $2850 \pm 617 \mathrm{~g}$ & $3010 \pm 782 \mathrm{~g}$ & $2986 \pm 899 \mathrm{~g}$ & NS \\
\hline$<2500 \mathrm{gm}$ & 18 & $3(30.0 \%)$ & $5(20.8 \%)$ & $10(28.6 \%)$ & NS \\
\hline$<1500 \mathrm{gm}$ & 5 & 0 & I (4.2\%) & 4 (II.4\%) & NS \\
\hline Vaginal delivery & 57 & $10(100 \%)$ & 19 (79.2\%) & $28(80.0 \%)$ & NS \\
\hline Home delivery & 5 & $3(30.0 \%)$ & $2(8.3 \%)$ & $0(0)$ & $0.005^{*}$ \\
\hline Fever & 62 & $10(100 \%)$ & 22 (9l.7\%) & $30(85.7 \%)$ & NS \\
\hline Death & 10 & $3(30 \%)$ & $3(12.5 \%)$ & 4 (II.4\%) & NS \\
\hline
\end{tabular}

Note: $* p<0.05$.

Abbreviation: NS, not significant.

from 30\% during 2001-2006 to $8.3 \%$ during 2007-2012, and no children with $E$. coli NM were born at home during 2013-2020 $(p<0.05)$.

\section{Antimicrobial Susceptibility of $E$. coli Neonatal Meningitis During 200I-2020}

The variation in the antibiotic susceptibility of all E. coli strains isolated from infants with $\mathrm{NM}$ in the three periods is presented in Figure 1. As shown in the figure, the overall susceptibility of $E$. coli to thirdgeneration cephalosporins (such as ceftazidime) decreased from $100 \%$ during 2001-2006 to 50\% during 2007-2012 and, subsequently, increased to approximately $71 \%$ during $2013-2020(p<0.05)$. This susceptibility pattern is closely related to the proportion of ESBL-producing bacteria, which increased from $0 \%$ during 2001-2006 to 50\% during 2007-2012 and decreased to $29 \%$ during $2013-2020(p<0.05)$. The susceptibility of $E$. coli to ampicillin decreased from $60.0 \%$ during 2001-2006 to $16.7 \%$ during 2007-2012, and subsequently increased to $33.3 \%$ during 2013-2020 $(p<0.05)$. There was no significant change in the susceptibility of $E$. coli strains to gentamicin (50\% during 2007-2012 compared with 59.3\% during 2013-2020). All E. coli strains in this study were susceptible to amikacin, cefoperazone-sulbactam, imipenem, and meropenem. The susceptibility to piperacillin-tazobactam and amoxicillin-clavulanic acid was very high $(95.8 \%$ and $91.7 \%$, respectively) during 2007-2012 and during 2013-2020 (96.7\% and 100\%, respectively). These results are similar to those reported in South Africa, where the susceptibility of E. coli to piperacillin-tazobactam was $93 \%{ }^{9}$

\section{Term and Premature Infants with E. coli Neonatal Meningitis}

Figure 2 shows the results of antibiotic susceptibility testing of meningitis-causing E. coli, comparing data from term and premature infants. The composition ratio of E. coli meningitis was significantly lower in premature infants $(24.6 \%, 17 / 69)$ than in term infants $(75.4 \%, 52 /$ 69) $(p<0.01)$. The susceptibility of $E$. coli isolated from premature infants with $E$. coli meningitis to ampicillin was $14.3 \%$, while that of term infants was $36.2 \%$, with no statistically significant difference. In isolates obtained from premature infants, the susceptibility of E. coli to levofloxacin was slightly lower than that in term infants ( $66.7 \%$ vs $79.6 \%, p=0.305$, not significant). Of the E. coli isolates obtained from premature infants, $60.0 \%$ were susceptible to third-generation cephalosporins (such as cefotaxime), at a slightly higher rate than isolates from term infants $(68.7 \%, p=0.530$, not significant). This trend is related to ESBL-producing E. coli, which accounts for $40.0 \%$ of all $E$. coli strains isolated from premature infants, compared with $30.0 \%$ of $E$. coli strains isolated from term infants $(p=0.639)$. In general, the majority of E. coli strains isolated from term and premature infants with meningitis in the study hospital were susceptible to amoxicillin-clavulanic acid, amikacin, cefoxitin, cefoperazone-sulbactam, imipenem, and meropenem.

\section{Distribution of E. coli Causing EOM and LOM}

Figure 3 shows the results of antibiotic susceptibility testing of E. coli causing meningitis-grouped by EOM vs LOM among neonates. Compared with that of E. coli isolated from infants with LOM, the susceptibility of 


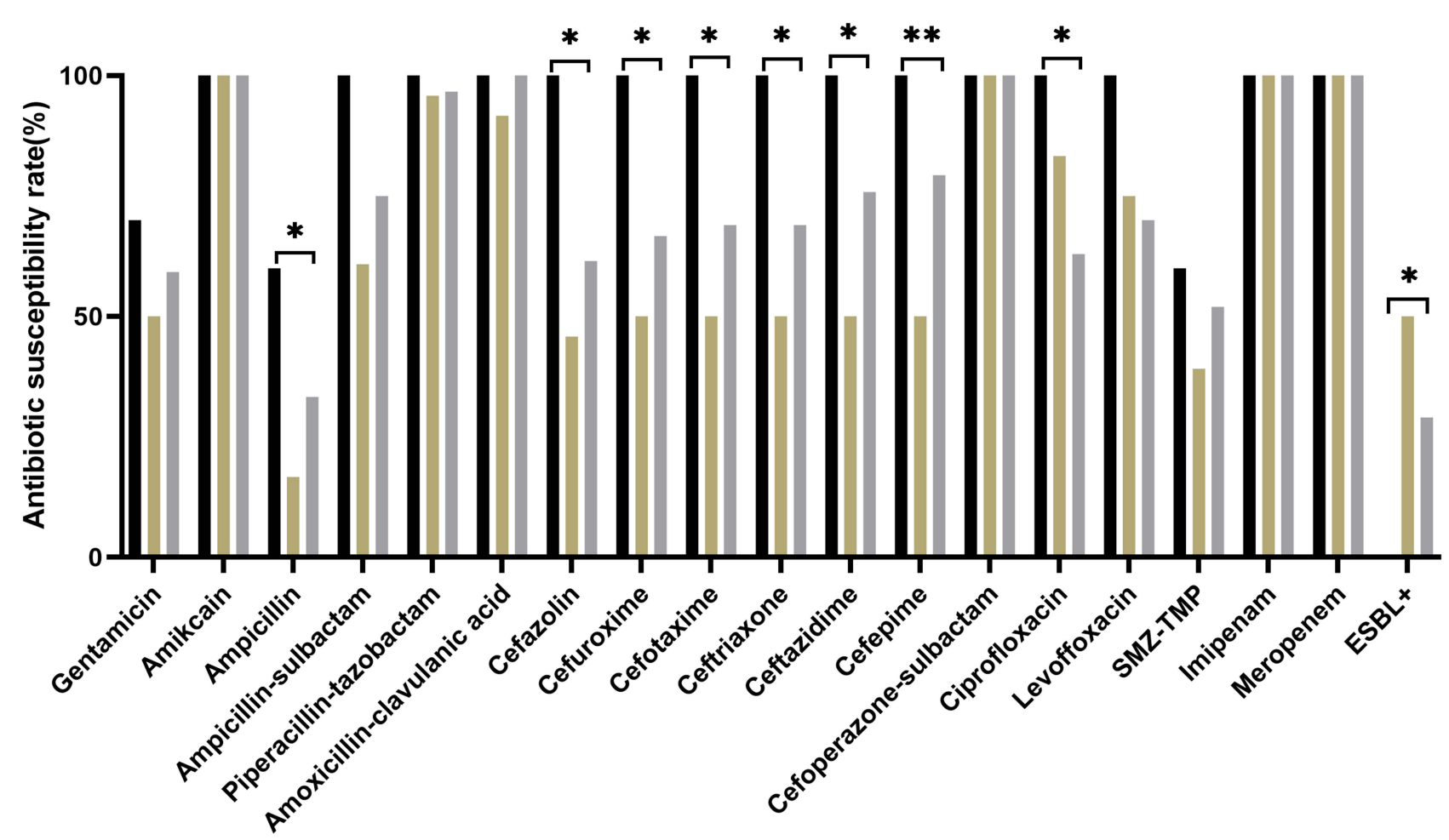

Figure I Antimicrobial susceptibility of all isolated $E$. coli in different periods were analyzed by Pearson's chi-squared test. $* P<0.05$. $* * P<0.0$ I.

E. coli isolated from infants with EOM to third-generation cephalosporins such as cefotaxime was significantly decreased $(91.7 \%$ vs $59.2 \%, p<0.05)$. This difference in drug susceptibility was primarily because of ESBLproducing $E$. coli, which accounts for $8.3 \%(1 / 12)$ of all E. coli isolates from children with EOM and $37.3 \%(20 /$ $53)$ of all E. coli isolates from children with LOM $(p<0.05)$.

\section{Discussion}

Neonates are at a high risk of meningitis, which might lead to neurologic complications. Severe neurodisability and milder motor and psychometric impairment result from NM. ${ }^{21}$ Despite global awareness of the risk factors for maternal and infantile infection and increased early treatment during the past 10 years, E. coli remains the primary causative organism of NM in developed countries. ${ }^{22-24}$ Large cohort studies have revealed that NM remains a substantial cause of sepsis-related morbidity and mortality in term and near-term infants. ${ }^{20}$ Bacterial resistance to commonly used antibiotics has become a global problem, ${ }^{25}$ and regional differences exist. ${ }^{15,26}$ When selecting empirical antibiotics, clinicians should consider local epidemiology (if known), early vs late disease onset, antimicrobial susceptibility patterns, and availability within resource constraints. ${ }^{27}$ Data regarding NM from developing countries are relatively scarce, and the bacterial profile may be quite different in these regions. We reviewed the current research in a large third-class hospital in eastern China over the last two decades. The findings show that the ratio of GBS meningitis has significantly increased, although E. coli persists as the main etiologic agent of NM in the hospital. The most common pathogens we identified, E. coli and GBS, are similar to those reported by Wiswell et al. ${ }^{28}$ A multicenter survey of neonatal purulent meningitis from 13 hospitals in northern China showed that $E$. coli was the most common pathogen of NM, with a rate of $21.1 \%$ among infants with $\mathrm{NM},{ }^{29}$ which is lower than that observed in our study $(37.9 \%$, 69/182). 


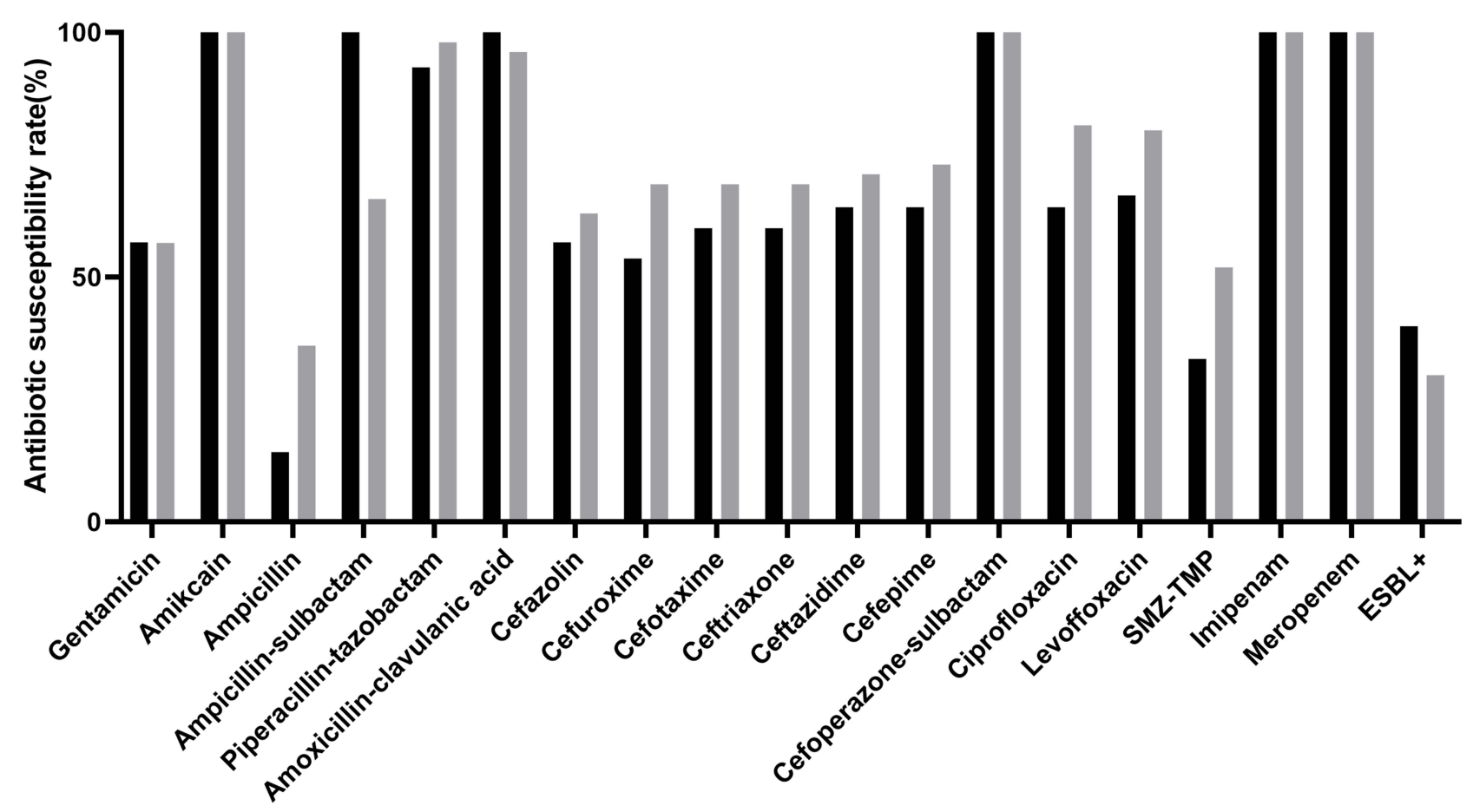

Figure 2 Antimicrobial susceptibility of all isolated $E$. coli from term and premature infants. There is no significant difference between the two groups.

In the past 10 years, the number of annual deliveries in our hospital has increased to approximately 10,000 births per year. This makes our center one of the largest perinatal centers in eastern China, where substantial socioeconomic changes have occurred owing to industrialization. In the last decade, more babies were born in hospitals, although some pregnant women delivered at home. Compared with that during 2001-2006, the proportion of children with E. coli meningitis who were born at home significantly decreased from 2007 to 2012, and there were no children with E. coli meningitis born at home from 2013 to 2020 $(3 / 10,2 / 24$, and $0 / 35$, respectively). E. coli frequently colonizes the maternal reproductive tract and can cause early neonatal infection. ${ }^{30,31}$ However, multidrug-resistant ESBL-producing bacteria are typically acquired from contaminated hospital environments and lead to increased risk of death, as observed in a large-scale sub-Saharan African study. ${ }^{9}$ Our results are consistent with these findings; all five children born at home during our study periods were term infants and were infected with ESBL-negative E. coli. The proportion of ESBL-producing E. coli isolated from infants with EOM was significantly lower than that of E. coli isolated from infants with LOM $(8.3 \%$ and $37.3 \%$, respectively).Notably, E. coli isolated from infants with EOM and from infants with LOM had significantly decreased antimicrobial susceptibility to third-generation cephalosporins, for example cefotaxime $(91.7 \%$ and $59.2 \%$, respectively). This may be owing to the ESBLproducing E. coli infection, which spreads in the community and contaminated hospital environments both vertically and horizontally through mobile genetic elements such as plasmids. A study revealed that infections with ESBL-producing E. coli spread frequently in households with babies, and improvement in community health was helpful to prevent the spread of ESBL-producing E. coli. ${ }^{32}$

The incidence of NM was between $0.12 \%$ and $1 \%$ in term infants and $3 \%$ in premature infants. ${ }^{13,33,34}$ A regional retrospective study conducted in Sweden during 1987-1996 estimated the incidence of NM at 0.3 per 1000 live births. ${ }^{35}$ This is in accordance with the results reported in the UK and Ireland, wherein the reported incidence was 0.38 per 1000 live births. ${ }^{36}$ The incidence of NM in developing countries was much higher at 0.8 6.1 per 1000 live births, with a mortality rate of $40-58 \%{ }^{15}$ 


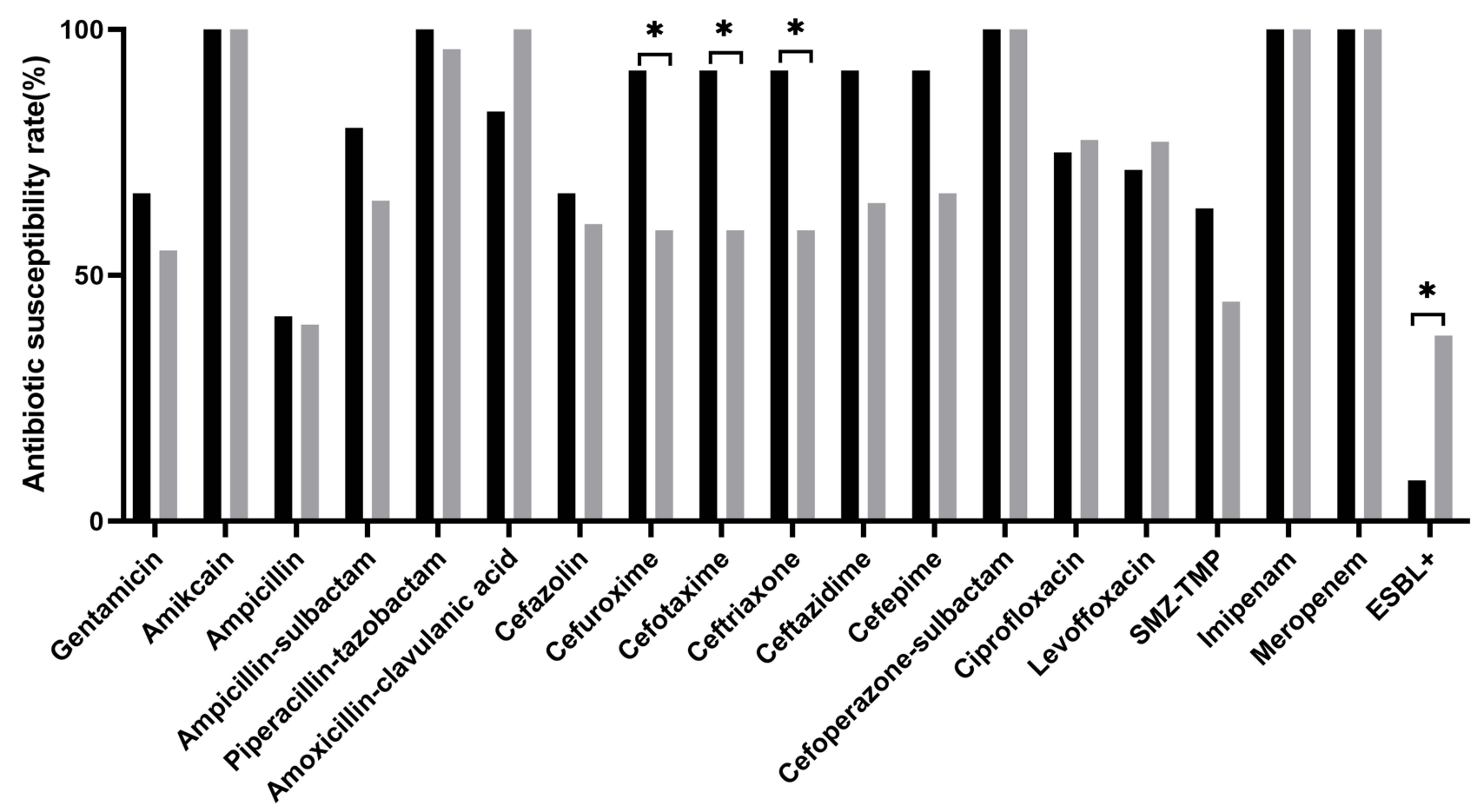

Figure 3 Antimicrobial susceptibility of all isolated E. coli from EOM and LOM. $* P<0.05$.

The incidence of culture-confirmed NM in our perinatal center increased from 0.12 per 1000 live-births during 2001-2006 to 0.26 per 1000 live-births during 20072012 and slightly decreased to 0.23 per 1000 live-births during 2013-2020. This rate is slightly lower than the rates reported for industrialized countries. This may be owing to the exclusion of some cases that had negative blood or CSF culture results despite having elevated CSF leukocyte counts $\left(\geq 20 \times 10^{6}\right.$ cells/L) and/or typical clinical manifestations of NM. In addition, we do not have information on polymerase chain reaction results which may lead to missed NM cases.

Neonatal E. coli causing meningitis is related to high rates of mortality and morbidity. ${ }^{37,38} \mathrm{~A}$ French national survey of 444 cases of neonatal bacterial meningitis from 2001 to 2007 reported a neonatal mortality rate of $13 \%$ for bacterial meningitis, with a mortality rate over twice that in premature infants $(26 \%)$ compared with term infants $(10 \%) ;{ }^{39}$ overall, the neonatal mortality rate for $E$. coli meningitis was $12 \%(15 / 123) .{ }^{39}$ In 2015 , it was reported that in Britain, the most common etiological agent of NM caused by gram-negative bacteria was $E$. coli $\mathrm{K} 1$, with a mortality rate of $10-15 \% .{ }^{40}$ As reported in Sweden in
2017, the pathogens with the highest NM mortality rate were the gram-negative bacteria Klebsiella pneumoniae $(33 \% ; 2 / 6)$ and E. coli $(11 \% ; 2 / 18) .{ }^{41}$ The case mortality rate of $E$. coli meningitis in our center remained relatively stable at $12.5 \%(3 / 24)$ during 2007-2012 and $11.4 \%(4 / 35)$ during 2013-2020, which is similar to the estimated mortality rate of $10 \%$ for NM in developed countries. ${ }^{15}$

For infected infants aged $<60$ days, the World Health Organization recommends using penicillins (such as ampicillin or penicillin) and aminoglycosides (such as gentamicin) or third-generation cephalosporins (such as ceftriaxone or cefotaxime). ${ }^{15}$ In the current study, only $16.7 \%$ of all $E$. coli isolates from infants with meningitis from 2007 to 2012 were susceptible to ampicillin, and $50 \%$ were susceptible to third-generation cephalosporins such as cefotaxime or ceftazidime. These rates were significantly lower than the susceptibility rates of $E$. coli isolated from 2001 to 2006, although the susceptibility of $E$. coli to ampicillin and cefotaxime or ceftazidime later increased from 2013 to 2020. Our data showed that approximately one-third $(21 / 65)$ of $E$. coli strains isolated from infants with NM in our NICU were multidrugresistant because of the production of ESBL. Although 
no ESBL-producing multidrug-resistant strains of E. coli were isolated from 2001 to 2006, an increasing number of ESBL-producing multidrug-resistant $E$. coli strains were isolated from 2007 to 2012, accounting for $50 \%$ of strains $(12 / 24)$. This increase may be due to the routine use of ceftriaxone in prenatal and neonatal infections, allowing for the development of antibiotic resistance to these drugs. Ceftriaxone has a wide antibacterial spectrum and is convenient to administer once daily. Fortunately, the number of ESBL-producing multidrug-resistant $E$. coli strains significantly decreased from 2013 to 2020, accounting for $29 \%$ of strains isolated from neonates with NM during this time period (9/31). This may be a result of China's strict control of antibiotic use over the past 10 years, particularly in children. New regulations restrict the use of antibiotics by requiring expert consensus on the diagnosis and management of neonatal sepsis. In our NICU, the management guidelines for antibiotic use are strictly implemented. Neonatologists adhere to guidelines for the indications, use, and discontinuation of antibacterial drugs in neonatal septicemia patients currently used in the United States. ${ }^{42}$ According to these guidelines, when antibiotics are used in children with high-risk factors, if infection symptoms (eg, fever, poor reaction, and feeding) are absent for $48 \mathrm{~h}$ and a blood culture produces no alarming results, then the use of antibiotics should be ceased immediately. For children with meningitis, penicillin combined with third-generation cephalosporins such as ceftriaxone is used empirically, and antibiotics are adjusted according to the drug sensitivity of the $E$. coli strain.

A study on neonatal septicemia and meningitis from 26 countries in Africa between 2008 and 2018 reported that the susceptibility of $E$. coli isolates from NM to ampicillin and gentamicin was $11 \%$ and $53 \%$, respectively. ${ }^{9}$ This value is higher than that reported from our center, in which $16.7 \%$ of $E$. coli isolates were susceptible to ampicillin from 2007 to 2012 and $33.3 \%$ from 2013 to 2020. During the 2013 to 2020 period, $59.3 \%$ of $E$. coli isolates were susceptible to gentamicin. Recently, the widespread use of carbapenems has caused a notable spread of carbapenem resistance. ${ }^{43}$ Despite this, all E. coli strains isolated from infants with meningitis in our NICU have remained susceptible to cefoperazone-sulbactam and carbapenem antibiotics.

Compared with that of term infants, the percentage of premature infants among E. coli $\mathrm{NM}$ cases has increased, and these infants are more susceptible to infections. A study from France has reported that E. coli is the most common cause of infection in late premature infants and very early premature infants. ${ }^{39}$ A prospective French survey collected the data from 325 children hospitalized globally with $E$. coli meningitis from 2001 to $2013{ }^{44}$ The results of this study showed that $65.2 \%$ of these children were born at term, $22.4 \%$ were late premature infants, and $12.5 \%$ were very early premature infants. ${ }^{44}$ Our results align with those from the French study. The proportions of term infants, late premature infants, and very premature or very early premature infants with $E$. coli meningitis were $75.4 \%(52 / 69)$, $11.6 \%(8 / 69)$, and $13 \%(9 / 69)$ of the total study population, respectively. Additionally, the proportion of premature infants with $E$. coli meningitis who had a birth weight of $<1500 \mathrm{~g}$ in our NICU increased from $4.2 \%$ to $11.4 \%$, which may be owing to the comprehensive actions of many factors. With the general progress of neonatal nursing and the improvement of NICU doctors' clinical skills, an increasing number of extremely premature infants have been successfully treated. In addition, this may be related to the finding that most maternal immunoglobulins do not cross the placenta before 32 weeks of gestation, and as a result, extremely premature infants are at a significantly higher risk of infections. ${ }^{45}$ Furthermore, early initiation of breastfeeding may confer protection against infections through the transfer of immunoglobulin A; however, breastfeeding is more common in term infants who are relatively less susceptible to infection. ${ }^{46}$

This study covered NM cases across 20 years in a single hospital. The study has its own limitations. First, the incidence of NM in term and premature infants was not calculated. Second, only confirmed cases before hospital discharge were analyzed. Further studies are needed to collect follow-up results regarding long-term neurologic morbidity and physical disability in these patients.

\section{Conclusion}

We studied the changes in the pattern of antibiotic susceptibility in NM-causing E. coli in a large perinatal medical center in Wenzhou located in eastern China. E. coli remains the major cause of neonatal bacterial meningitis, despite the increase in GBS-caused NM. The proportion of ESBL-producing multi-drug-resistant $E$. coli in NM isolates has significantly decreased in the last 8 years owing to compliance with strict guidelines concerning the use of antibiotics. Therefore, third-generation antibiotic cephalosporins are still effective for treating E. coli NM. In our center, ceftriaxone and penicillin were empirical choices of antimicrobial therapy for NM before the etiology was determined. Third-generation cephalosporins and 
penicillin are the first option for treating NM in developing countries as these antibiotics can treat infection caused by E. coli and GBS. Continuous monitoring of antibiotic susceptibility in NM isolates is necessary to ensure the effectiveness of the standard empirical treatment and to monitor the development of antibiotic resistance. Our study will be helpful in selecting more appropriate antibiotics for empirical treatment in developing countries with similar bacterial spectra and sensitivities.

\section{Author Contributions}

All authors made substantial contributions to conception and design, acquisition of data, or analysis and interpretation of data; took part in drafting the article or revising it critically for important intellectual content; agreed to submit to the current journal; gave final approval of the version to be published; and agree to be accountable for all aspects of the work.

\section{Funding}

This research was funded by Wenzhou Municipal Science \& Technology Bureau (No. 2020Y0470).

\section{Disclosure}

There are no conflicts of interest to declare.

\section{References}

1. Heath P, Nik Yusoff N, Baker C. Neonatal meningitis. Arch Dis Child Fetal Neonatal Ed. 2003;88:F173-178. doi:10.1136/fn.88.3.f173

2. Pelkonen T, Urtti S, Dos Anjos E, et al. Aetiology of bacterial meningitis in infants aged $<90$ days: prospective surveillance in Luanda, Angola. Int $J$ Infect Dis. 2020;97:251-257. doi:10.1016/j.ijid.2020. 06.016

3. Houdouin V, Bonacorsi S, Bidet P, et al. Association between mortality of Escherichia coli meningitis in young infants and non-virulent clonal groups of strains. Clin Microbiol Infect. 2008;14:685-690. doi:10.1111/j.1469-0691.2008.02019.x

4. Holt D, Halket S, de Louvois J, Harvey D. Neonatal meningitis in England and Wales: 10 years on. Arch Dis Child. 2001;84:F85-89. doi:10.1136/fn.84.2.f85

5. Huang W, Lin CY, Hashimoto M, et al. The role of the bacterial protease Prc in the uropathogenesis of extraintestinal pathogenic Escherichia coli. J Biomed Sci. 2020;27:14. doi:10.1186/s12929-0190605-y

6. Kennedy C, Walsh C, Karczmarczyk M, et al. Multi-drug resistant Escherichia coli in diarrhoeagenic foals: pulsotyping, phylotyping, serotyping, antibiotic resistance and virulence profiling. Vet Microbiol. 2018;223:144-152. doi:10.1016/j.vetmic.2018.08.009

7. Allocati N, Masulli M, Alexeyev M, Di Ilio C. Escherichia coli in Europe: an overview. Int $J$ Environ Res Public Health. 2013;10:6235-6254. doi:10.3390/ijerph10126235

8. Bekker V, Bijlsma M, van de Beek D, Kuijpers T, van der Ende A. Incidence of invasive group B streptococcal disease and pathogen genotype distribution in newborn babies in the Netherlands over 25 years: a nationwide surveillance study. Lancet Infect Dis. 2014;14:1083-1089. doi:10.1016/s1473-3099(14)70919-3
9. Okomo U, Akpalu EN, Le Doare K, et al. Aetiology of invasive bacterial infection and antimicrobial resistance in neonates in sub-Saharan Africa: a systematic review and meta-analysis in line with the STROBE-NI reporting guidelines. Lancet Infect Dis. 2019;19:1219-1234. doi:10.1016/s1473-3099(19)30414-1

10. Liu G, He S, Zhu X, Li Z. Early onset neonatal bacterial meningitis in term infants: the clinical features, perinatal conditions, and in-hospital outcomes: a single center retrospective analysis. Medicine. 2020;99:e22748. doi:10.1097/md.000000000 0022748

11. Stoll B, Hansen NI, Sanchez PJ, et al. Early onset neonatal sepsis: the burden of group B Streptococcal and E. coli disease continues. Pediatrics. 2011;127:817-826. doi:10.1542/peds.2010-2217

12. de Louvois J, Blackbourn J, Hurley R, Harvey D. Infantile meningitis in England and Wales: a two year study. Arch Dis Child. 1991;66:603-607. doi:10.1136/adc.66.5.603

13. de Louvois J, Halket S, Harvey D. Neonatal meningitis in England and Wales: sequelae at 5 years of age. Eur $J$ Pediatr. 2005; 164:730-734. doi:10.1007/s00431-005-1747-3

14. Zhao Z, Yu J, Zhang H, Li J, Li Z. Five-year multicenter study of clinical tests of neonatal purulent meningitis. Clin Pediatr (Phila). 2018;57:389-397. doi:10.1177/0009922817728699

15. Furyk J, Swann O, Molyneux E. Systematic review: neonatal meningitis in the developing world. Trop Med Int Health. 2011;16:672-679. doi:10.1111/j.1365-3156.2011.02750.x

16. Berardi A, Sforza F, Baroni L, et al. Epidemiology and complications of late-onset sepsis: an Italian area-based study. PLoS One. 2019;14: e0225407. doi:10.1371/journal.pone.0225407

17. El-Naggar W, Afifi J, McMillan D, et al. Epidemiology of meningitis in canadian neonatal intensive care units. Pediatr Infect Dis J. 2019;38:476-480. doi:10.1097/inf.0000000000002247

18. Zhu ML, Hu QH, Mai JY, et al. Analysis of pathogenic bacteria and drug resistance in neonatal purulent meningitis. Chin $J$ Pediatr. 2015;53:51-56.

19. Chen ZQ, Zhou D, Lin FF. Comparative analysis of clinical characteristics of neonates with purulent meningitis caused by different pathogens. China Modern Med. 2020;27:13-17.

20. Garges H, Moody MA, Cotten CM, et al. Neonatal meningitis: what is the correlation among cerebrospinal fluid cultures, blood cultures, and cerebrospinal fluid parameters? Pediatrics. 2006;117:1094-1100. doi:10.1542/peds.2005-1132

21. Stevens J, Eames M, Kent A, Halket S, Holt D, Harvey D. Long term outcome of neonatal meningitis. Arch Dis Child. 2003;88:F179-184. doi:10.1136/fn.88.3.f179

22. Bonacorsi S, Bingen E. Molecular epidemiology of Escherichia coli causing neonatal meningitis. IJMM. 2005;295:373-381. doi:10.1016/ j.ijmm.2005.07.011

23. Stoll B, Hansen N, Fanaroff AA, et al. Late-onset sepsis in very low birth weight neonates: the experience of the NICHD Neonatal Research Network. Pediatrics. 2002;110:285-291. doi:10.1542/ peds.110.2.285

24. Wang M, Kim K. Cytotoxic necrotizing factor 1 contributes to Escherichia coli meningitis. Toxins. 2013;5:2270-2280. doi:10. $3390 /$ toxins 5112270

25. Vergnano S, Sharland M, Kazembe P, Mwansambo C, Heath P. Neonatal sepsis: an international perspective. Arch Dis Child Fetal Neonatal Ed. 2005;90:F220-224. doi:10.1136/adc.2002.022863

26. Osrin D, Vergnano S, Costello A. Serious bacterial infections in newborn infants in developing countries. Curr Opin Infect Dis. 2004;17:217-224. doi:10.1097/00001432-200406000-00008

27. Odio C. Cefotaxime for treatment of neonatal sepsis and meningitis. Diagn Microbiol Infect Dis. 1995;22:111-117. doi:10.1016/07328893(95)00093-p

28. Wiswell T, Baumgart S, Gannon C, Spitzer A. No lumbar puncture in the evaluation for early neonatal sepsis: will meningitis be missed? Pediatrics. 1995;95:803-806. 
29. Liu C. Neonatal bacterial meningitis in 13 hospitals of Northern China: epidemiology, etiology and outcomes. Chin J Neonatol. 2017;32:415-419.

30. Kerur B, Vishnu Bhat B, Harish B, Habeebullah S, Uday Kumar C. Maternal genital bacteria and surface colonization in early neonatal sepsis. Indian J Pediatr. 2006;73:29-32. doi:10. 1007/bf02758256

31. Soto S, Bosch J, Jimenez de Anta M, Vila J. Comparative study of virulence traits of Escherichia coli clinical isolates causing early and late neonatal sepsis. J Clin Microbiol. 2008;46:1123-1125. doi:10.1128/jcm.01682-07

32. Kardaś-Słoma L, Yazdanpanah Y, Perozziello A, et al. Hand hygiene improvement or antibiotic restriction to control the household transmission of extended-spectrum $\beta$-lactamaseproducing Escherichia coli: a mathematical modelling study. Antimicrob Resist Infect Control. 2020;9:139. doi:10.1186/ s13756-020-00803-9

33. Safavieh M, Ahmed M, Tolba M, Zourob M. Microfluidic electrochemical assay for rapid detection and quantification of Escherichia coli. Biosens Bioelectron. 2012;31:523-528. doi:10.1016/j.bios.20 11.11.032

34. Friedrich T, Rahmann S, Weigel W, et al. High-throughput microarray technology in diagnostics of enterobacteria based on genome-wide probe selection and regression analysis. BMC Genom. 2010;11:591. doi:10.1186/1471-2164-11-591

35. Persson E, Trollfors B, Brandberg L, Tessin I. Septicaemia and meningitis in neonates and during early infancy in the Göteborg area of Sweden. Acta Paediatr. 2002;91:1087-1092. doi:10.1080/ 080352502760311593

36. Okike I, Johnson AP, Henderson KL, et al. Incidence, etiology, and outcome of bacterial meningitis in infants aged $<90$ days in the United kingdom and Republic of Ireland: prospective, enhanced, national population-based surveillance. Clin Infect Dis. 2014;59: e150-157. doi:10.1093/cid/ciu514
37. Zhu N, Liu W, Prakash A, Zhang C, Kim K. Targeting E. coli invasion of the blood-brain barrier for investigating the pathogenesis and therapeutic development of E. coli meningitis. Cell Microbiol. 2020;22:e13231. doi:10.1111/cmi.13231

38. Kim B, Kang J, Kim K. Invasion processes of pathogenic Escherichia coli. IJMM. 2005;295:463-470. doi:10.1016/j.ijmm.2005.07.004

39. Gaschignard J, Levy C, Romain O, et al. Neonatal bacterial meningitis: 444 cases in 7 years. Pediatr Infect Dis J. 2011;30:212-217. doi:10.1097/inf.0b013e3181 fab1e7

40. Alkeskas A, Ogrodzki P, Saad M, et al. The molecular characterisation of Escherichia coli $\mathrm{K} 1$ isolated from neonatal nasogastric feeding tubes. BMC Infect Dis. 2015;15:449. doi:10.1186/s12879-015-1210-7

41. Johansson Gudjónsdóttir M, Elfvin A, Hentz E, et al. Changes in incidence and etiology of early-onset neonatal infections 1997-2017 a retrospective cohort study in western Sweden. BMC Pediatr. 2019;19:490. doi:10.1186/s12887-019-1866-z

42. Polin R. Management of neonates with suspected or proven early-onset bacterial sepsis. Pediatrics. 2012;129:1006-1015. doi:10. 1542/peds.2012-0541

43. Wang F, Jiang J, Shi G, Wang J, Zhou S. Klebsiella pneumoniaeAnti-infective treatment of purulent meningitis caused by carbapenem-resistant in a newborn: a case report. Transl Pediatr. 2020;9:713-719. doi:10.21037/tp-20-296

44. Basmaci R, Bonacorsi S, Bidet P, et al. Escherichia coli meningitis features in 325 children from 2001 to 2013 in France. Clin Infect Dis. 2015;61:779-786. doi:10.1093/cid/civ367

45. Heath PT, Okike IO. Neonatal bacterial meningitis: an update. Paediatr Child Health. 2010;20:526-530. doi:10.1016/j.paed.20 10.04.001

46. Debes A, Kohli A, Walker N, Edmond K, Mullany L. Time to initiation of breastfeeding and neonatal mortality and morbidity: a systematic review. BMC Public Health. 2013;13:S19. doi:10.15 42/peds.2010-2217
International Journal of General Medicine

\section{Publish your work in this journal}

The International Journal of General Medicine is an international, peer-reviewed open-access journal that focuses on general and internal medicine, pathogenesis, epidemiology, diagnosis, monitoring and treatment protocols. The journal is characterized by the rapid reporting of reviews, original research and clinical studies

\section{Dovepress}

across all disease areas. The manuscript management system is completely online and includes a very quick and fair peer-review system, which is all easy to use. Visit http://www.dovepress.com/ testimonials.php to read real quotes from published authors. 